\title{
ON PARAMETERIZATIONS OF RATIONAL ORBITS OF SOME FORMS OF PREHOMOGENEOUS VECTOR SPACES
}

\author{
TAKASHI TANIGUCHI
}

\begin{abstract}
In this paper, we consider the structure of rational orbit of some prehomogeneous vector spaces originally motivated by Wright and Yukie. After we give a refinement of WrightYukie's construction, we apply it to the inner forms of the $D_{5}$ and $E_{7}$ types. Parameterizations of reducible algebras for the cubic cases are included.
\end{abstract}

\section{INTRODUCTION}

The aim of paper is to give a complement of the series of the work [WY92, KY97, Y97b] by Yukie, Wright and Kable on parameterizing separable algebras of degree 2, 3, 4 and 5 by means of non-degenerate rational orbits of certain prehomogeneous vector spaces. The prehomogeneous vector space we treat mainly in this paper are inner forms of what are called $D_{5}$ and $E_{7}$ type. We also consider the parameterizations of "reducible algebras" for the cubic cases $\left(G_{2}, E_{6}\right.$ and $E_{7}$ type.)

Let $k$ be a field of any characteristic and $\mathcal{B}$ a quaternion algebra over $k$ with the canonical involution $*$. We denote by $\mathcal{N}$ the reduced norm. Let $\mathrm{M}_{n}(\mathcal{B})$ be the set of $n$ by $n$ matrices with entries in $\mathcal{B}$. This algebra has an involution $x=\left(x_{i j}\right) \mapsto x^{\iota}=\left(x_{j i}^{*}\right)$. Let $\mathrm{H}_{n}(\mathcal{B})=\left\{x \in \mathrm{M}_{n}(\mathcal{B}) \mid\right.$ $\left.x^{\iota}=x\right\}$. Then $\mathrm{GL}_{n}(\mathcal{B})$ acts on this space via $(g, x) \mapsto g \cdot x=g x g^{\iota}$ for $g \in \mathrm{GL}_{n}(\mathcal{B}), x \in \mathrm{H}_{n}(\mathcal{B})$. In this paper we consider the representation

$$
G=\mathrm{GL}_{n}(\mathcal{B}) \times \mathrm{GL}_{2}(k), \quad V=\mathrm{H}_{n}(\mathcal{B}) \otimes k^{2}, \quad(n=2,3 .)
$$

We regard this as a representation of an algebraic group $G$ defined over $k$. This is an inner form of the prehomogeneous vector space $\left(\mathrm{GL}_{2 n} \times \mathrm{GL}_{2}, \wedge^{2} k^{2 n} \otimes k^{2}\right)$ and in the case $\mathcal{B} \cong \mathrm{M}_{2}(k)$ they are equivalent. These are referred to as the $D_{5}$ type (resp. $E_{7}$ type) if $n=2$ (resp. $n=3$.) There is a non-zero polynomial $P$ in $V$ such that $P(g x)=\chi(g) P(x)$ for some nontrivial character $\chi$ on $G$. We put $V^{\mathrm{ss}}=\{x \in V \mid P(x) \neq 0\}$. Let $\mathcal{A}_{n}$ be the set of isomorphism classes of separable $k$-algebras of degree $n$. In Section 3 we prove the following.

Theorem 1.1 (Theorem 3.15, Theorem 3.16). For $n=2,3$, there exist the canonical surjective map $\Psi: G(k) \backslash V^{\mathrm{ss}}(k) \ni x \mapsto L_{x} \in \mathcal{A}_{n}$ such that the followings hold.

(1) For $n=2, \Psi$ is bijective. Also for $x \in V^{\mathrm{ss}}(k)$, the identity component of the stabilizer $G_{x}^{\circ}$ is isomorphic to $\left(\mathcal{B} \otimes L_{x}\right)^{\times}$as an algebraic group over $k$.

(2) For $n=3$, the fiber of $L \in \mathcal{A}_{3}$ is parameterized by the set

$$
\left(k^{\times} \cdot \operatorname{Im}\left(\mathcal{N}:(\mathcal{B} \otimes L)^{\times} \rightarrow L^{\times}\right)\right) \backslash L^{\times} / \operatorname{Aut}_{k}(L) .
$$

For $n=3$, we also determine the structure of $G_{x}^{\circ}$ in Theorem 3.16 and the explicit correspondence of the fibers of $\Psi$ in Theorem 3.17. We note that (1.1) and representations in [KY97] numbered as (1) and (3) have something in common, and results similar to Theorem 1.1 have been obtained for those cases. After Wright-Yukie [WY92] considered 8 prehomogeneous vector spaces, some of their $k$-forms were studied by Kable-Yukie [KY97] and by the author

Date: September 15, 2018.

Key words and phrases. Prehomogeneous vector space, Galois cohomology.

Financial support is provided by Research Fellowships for Young Scientists of Japan Society for the Promotion of Science. 
[T04], and this paper almost covers the remaining $k$-forms of them. For the classification of $k$-forms of prehomogeneous vector spaces, see H. Saito's paper [Sa97]. We briefly discuss the connection of our results and H. Saito's decomposition formula [Sa99] of the global zeta functions of prehomogeneous vector spaces at the end of Section 3.

We follow the argument of [WY92, KY97], but we give a refinement. Let $(G, V)$ be a prehomogeneous vector space treated in [WY92] or [KY97] parameterizing $\mathcal{A}_{i}$. They constructed the map $G(k) \backslash V^{\mathrm{ss}}(k) \rightarrow \mathcal{A}_{i}$ in two ways, via geometry and cohomology and then showed that two constructions coincide by means of case by case computation. In this paper we interpret the cohomology theoretic construction more intrinsically so that it naturally coincides with the geometric construction. We see this in Theorem 3.8. This also simplify the study of the fiber structure of $\Psi$. We write the proof for the representation (1.1) but the arguments are parallel for cases those treated in [WY92, KY97, T04]. For this see Remark 3.10.

Another topic we consider in this paper is on parameterizations of reducible algebras. Let $\mathcal{E}_{n} \subset \mathcal{A}_{n}$ be the subset consisting of degree $n$ field extensions of $k$. From number theoretical interests, we often would like to understand the set $\mathcal{E}_{n}$ rather than $\mathcal{A}_{n}$. For that purpose, it is sometimes useful to parameterize the set of reducible algebras $\mathcal{A}_{n} \backslash \mathcal{E}_{n}$ via linear representations. For the case of the space of binary cubic forms $\left(G_{2}\right.$ type) this was done by Shintani [Sh75]. In Section 4 we will consider for other cubic cases i.e., $E_{6}$ and $E_{7}$ types. We hope this result is useful for studying the theory of zeta functions and some other directions.

This paper is organized as follows. In Section 2 we review the definition and basic properties of the non-abelian Galois cohomology. In Section 3 we first give the compatibility of geometric parameterization and cohomological parameterization. We refine the argument of [WY92] and simplify the description of the fiber structure. After that we treat $D_{5}$ and $E_{7}$ type prehomogeneous vector spaces explicitly. A discussion of a decomposition of the global zeta function is given at the end of the section. In Section 4 we consider the parameterizations of reducible algebras for the cubic cases.

Notation. Throughout this paper $k$ is a field of any characteristic. We fix a separable closure $k^{\mathrm{s}}$ of $k$ and regard any field extensions of $k$ as subfields of $k^{\mathrm{s}}$. Let $\Gamma=\operatorname{Gal}\left(k^{\mathrm{s}} / k\right)$. We assume $\Gamma$ acts on $k^{\text {s }}$ from the right. Let $X$ be a scheme over $k$. For a $k$-algebra $R$, the set of $R$-rational points is denoted by $X(R)$ and the base change of $X$ to a scheme over $R$ is denoted by $X \times_{k} R$ or simply $X_{R}$. We write $X^{\mathrm{s}}=X \times_{k} k^{\mathrm{s}}$.

For a finite separable field extension $L / k$ and a scheme $Y$ over $L$, we denote by $\mathcal{R}_{L / k}(Y)$ the restriction of scalars. Let $L=L_{1} \times \cdots \times L_{n}$ where each $L_{i} / k$ is a finite separable field extension and $X$ a scheme over $k$. We define a scheme $\mathcal{R}_{L / k}\left(X_{L}\right)$ over $k$ by

$$
\mathcal{R}_{L / k}\left(X_{L}\right)=\mathcal{R}_{L_{1} / k}\left(X_{L_{1}}\right) \times \cdots \times \mathcal{R}_{L_{n} / k}\left(X_{L_{n}}\right)
$$

This scheme represents the functor $R \mapsto X(L \otimes R)$ where $R$ is any $k$-algebra.

\section{Review of Galois cohomology}

In this section we recall the definition of Galois cohomology and review its properties we use in this paper. Most of the proofs are omitted. For details, Serre's book [Se65, Chapter I $\S 5$, Chapter III $\S 1]$. We first recall the definition of the Galois cohomology and properties of sequences.

Definition 2.1. Let $G$ be a group scheme over $k$. We consider the discrete topology on $G\left(k^{\mathrm{s}}\right)$. A continuous function $h: \Gamma \rightarrow G\left(k^{\mathrm{s}}\right)$ is called a 1-cocycle if $h(\sigma \tau)=h(\tau) h(\sigma)^{\tau}$ for all $\sigma, \tau \in \Gamma$. Two 1-cocycles $h_{1}$ and $h_{2}$ are equivalent if there exists $g \in G\left(k^{\mathrm{s}}\right)$ such that $h_{2}(\sigma)=g^{-1} h_{1}(\sigma) g^{\sigma}$ for all $\sigma$. The set of equivalence classes of 1-cocycles is denoted by $\mathrm{H}^{1}(k, G)$ and called the first Galois cohomology set of $G$. The class of the trivial 1-cocycle $\Gamma \ni \sigma \mapsto 1 \in G\left(k^{\mathrm{s}}\right)$ (where 1 is the identity) is called the trivial class. 
By definition, $\mathrm{H}^{1}\left(k, G_{1} \times G_{2}\right)=\mathrm{H}^{1}\left(k, G_{1}\right) \times \mathrm{H}^{1}\left(k, G_{2}\right)$. When $G$ is not commutative, $\mathrm{H}^{1}(k, G)$ does not have a group structure in general. But by regarding the trivial class as its base point, $\mathrm{H}^{1}(k, G)$ becomes a pointed set. If $G_{1} \rightarrow G_{2}$ is a homomorphism of group schemes over $k$, it obviously induces a homomorphism $\mathrm{H}^{1}\left(k, G_{1}\right) \rightarrow \mathrm{H}^{1}\left(k, G_{2}\right)$ of pointed sets. For the rest of this paper we may use the notation $h=\left(h_{\sigma}\right)_{\sigma \in \Gamma}$ for a 1-cocycle, where $h_{\sigma}$ stands for the value of $h$ at $\sigma \in \Gamma$.

Let $G_{1}, G_{2}$ and $G_{3}$ be algebraic groups over $k$. We say a sequence

$$
1 \longrightarrow G_{1} \stackrel{\phi_{1}}{\longrightarrow} G_{2} \stackrel{\phi_{2}}{\longrightarrow} G_{3} \longrightarrow 1
$$

is exact if $G_{1}$ is a normal subgroup of $G_{2}$ by $\phi_{1}, \operatorname{ker}\left(\phi_{2}\right)=G_{1}$, and $G_{2}\left(k^{\mathrm{s}}\right) \rightarrow G_{3}\left(k^{\mathrm{s}}\right)$ is surjective. In this situation $G_{3}(k)$ acts on $\mathrm{H}^{1}\left(k, G_{1}\right)$ from the right. We recall its definition. Suppose $g \in G_{3}(k)$ and $\bar{h} \in \mathrm{H}^{1}\left(k, G_{1}\right)$. Let $h=\left(h_{\sigma}\right)$ be a 1 -cocycle in the class $\bar{h}$. Take $\tilde{g} \in G_{2}\left(k^{\mathrm{s}}\right)$ such that $\phi_{2}(\tilde{g})=g$. Then we see that $h_{\sigma}^{\prime}=\tilde{g}^{-1} h_{\sigma} \tilde{g}^{\sigma}$ is in $G_{1}\left(k^{\mathrm{s}}\right)$ for all $\sigma \in \Gamma$ and $h^{\prime}=\left(h_{\sigma}^{\prime}\right)$ is a 1-cocycle of $G_{1}$. It is easy to see that the class $\overline{h^{\prime}}$ of $h^{\prime}$ does not depend on the choice of $\tilde{g}$ and $h$, and $(g, \bar{h}) \mapsto \overline{h^{\prime}}$ defines a right action of $G_{3}(k)$. By considering the action of $G_{3}(k)$ on the trivial element in $\mathrm{H}^{1}\left(k, G_{1}\right)$, we get a map $G_{3}(k) \rightarrow \mathrm{H}^{1}\left(k, G_{1}\right)$.

Theorem 2.2. If (2.1) is exact, then the sequence

$$
1 \longrightarrow G_{1}(k) \longrightarrow G_{2}(k) \longrightarrow G_{3}(k) \longrightarrow \mathrm{H}^{1}\left(k, G_{1}\right) \longrightarrow \mathrm{H}^{1}\left(k, G_{2}\right) \longrightarrow \mathrm{H}^{1}\left(k, G_{3}\right)
$$

is also exact. Moreover, the map $\mathrm{H}^{1}\left(k, G_{1}\right) / G_{3}(k) \rightarrow \mathrm{H}^{1}\left(k, G_{2}\right)$ is injective.

Note that the exactness in the theorem above means that the classes which map to the trivial class come from the previous set.

Let $G$ be an affine algebraic group and $H$ a closed subgroup. Then it is known that the geometric quotient $X=G / H$ exists. The scheme $X$ does not have a group structure in general but in the case the injection $G\left(k^{\mathrm{s}}\right) / H\left(k^{\mathrm{s}}\right) \rightarrow X\left(k^{\mathrm{s}}\right)$ is surjective also, we can still define the map $X(k) \rightarrow \mathrm{H}^{1}(k, H)$ the same way as above. That is, for $x \in X(k)$, take $g \in G\left(k^{\mathrm{s}}\right)$ that goes to $x$ by the quotient map and then we map $x$ to a cohomology class of the 1-cocycle $\left(g^{-1} g^{\sigma}\right)_{\sigma \in \Gamma}$. For this map the following holds.

Theorem 2.3. Assume $G\left(k^{\mathrm{s}}\right) / H\left(k^{\mathrm{s}}\right) \rightarrow X\left(k^{\mathrm{s}}\right)$ is bijective. Then $G(k) \backslash X(k) \rightarrow \mathrm{H}^{1}(k, H)$ is injective and

$$
G(k) \backslash X(k) \longrightarrow \mathrm{H}^{1}(k, H) \longrightarrow \mathrm{H}^{1}(k, G)
$$

is exact.

We next recall how the Galois cohomology classifies $k$-forms of a given algebraic object. Let $X$ be a variety over $k$. (We do not assume $X$ is connected.) Then $\Gamma$ acts on $X^{\mathrm{s}}$ from the right. For $\sigma \in \Gamma$ we denote the induced action $X^{\mathrm{s}} \rightarrow X^{\mathrm{s}}$ by $\sigma_{X}$;

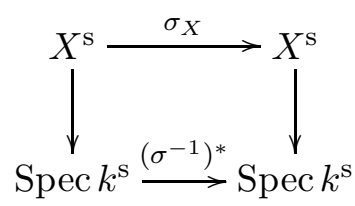

Let $\operatorname{Aut}(X)$ be the automorphism group of $X$. We assume Aut $(X)$ acts on $X$ from the right. It is known that Aut $(X)$ is a group scheme over $k$ and its $R$-rational points $\operatorname{Aut}(X)(R)$ is the group of $R$-automorphism of $X \times_{k} R$. Hence $\Gamma$ acts on $\operatorname{Aut}(X)\left(k^{\mathrm{s}}\right)=\operatorname{Aut}_{k^{\mathrm{s}}}\left(X^{\mathrm{s}}\right)$ from the right. For $\alpha \in \operatorname{Aut}_{k^{\mathrm{s}}}\left(X^{\mathrm{s}}\right)$ and $\sigma \in \Gamma$, this action is given by

$$
\alpha^{\sigma}=\sigma_{X} \circ \alpha \circ \sigma_{X}^{-1} ; \quad X^{\mathrm{s}} \stackrel{\sigma_{X}^{-1}}{\longrightarrow} X^{\mathrm{s}} \stackrel{\alpha}{\longrightarrow} X^{\mathrm{s}} \stackrel{\sigma_{X}}{\longrightarrow} X^{\mathrm{s}} .
$$

Definition 2.4. A variety $Y$ over $k$ is called a $k$-form of $X$ if $Y^{\mathrm{s}}$ is $k^{\mathrm{s}}$-isomorphic to $X^{\mathrm{s}}$. 
Let $Y$ be a $k$-form of $X$. Take a $k^{\mathrm{s}}$-isomorphism $\psi: Y^{\mathrm{s}} \rightarrow X^{\mathrm{s}}$. For $\sigma \in \Gamma$, let

$$
h_{Y}(\sigma)=\sigma_{X} \circ \psi \circ \sigma_{Y}^{-1} \circ \psi^{-1} ; \quad X^{\mathrm{s}} \stackrel{\psi^{-1}}{\longrightarrow} Y^{\mathrm{s}} \stackrel{\sigma_{Y}^{-1}}{\longrightarrow} Y^{\mathrm{s}} \stackrel{\psi}{\longrightarrow} X^{\mathrm{s}} \stackrel{\sigma_{X}}{\longrightarrow} X^{\mathrm{s}},
$$

which is an element of $\operatorname{Aut}(X)\left(k^{\mathrm{s}}\right)=\operatorname{Aut}_{k^{\mathrm{s}}}\left(X^{\mathrm{s}}\right)$. Then since

$$
\begin{aligned}
h_{Y}(\tau) h_{Y}(\sigma)^{\tau} & =h_{Y}(\sigma)^{\tau} \circ h_{Y}(\tau)=\tau_{X} \circ h_{Y}(\sigma) \circ \tau_{X}^{-1} \circ h_{Y}(\tau) \\
& =\tau_{X} \circ \sigma_{X} \circ \psi \circ \sigma_{Y}^{-1} \circ \psi^{-1} \circ \tau_{X}^{-1} \circ \tau_{X} \circ \psi \circ \tau_{Y}^{-1} \circ \psi^{-1} \\
& =(\sigma \tau)_{X} \circ \psi \circ(\sigma \tau)_{Y}^{-1} \circ \psi^{-1} \\
& =h_{Y}(\sigma \tau),
\end{aligned}
$$

$h_{Y}$ is a 1-cocycle of Aut $(X)$. It is easy to see that its cohomology class does not depend on the choice of $\psi$. We also denote the cohomology class by $h_{Y}$. The following lemma is easy to prove.

Lemma 2.5. By associating $h_{Y}$ to a $k$-form $Y$ of $X$, we have an injective map from the set of isomorphism classes of $k$-forms of $X$ to $\mathrm{H}^{1}(k$, Aut $(X))$. In this correspondence, the isomorphism class of $X$ goes to the trivial class.

In Section 3 we use the following theorem.

Theorem 2.6. If $X$ is quasi-projective as a variety (not necessary connected), then the map above is bijective.

The following basic result is useful for the concrete calculation in Section 3.

Proposition 2.7. (1) Let $A$ be a finite dimensional simple algebra over $k$. We regard $A^{\times}$as an algebraic group over $k$. Then $\mathrm{H}^{1}\left(k, A^{\times}\right)=\{1\}$.

(2) Let $L / k$ be a finite separable field extension and $G$ a connected algebraic group over $L$. Then $\mathrm{H}^{1}\left(k, \mathcal{R}_{L / k}(G)\right) \cong \mathrm{H}^{1}(L, G)$.

\section{Rational orbits of inner forms of $D_{5}, E_{7}$ TYPeS}

3.1. A general construction. Let $\mathcal{B}$ be a quaternion algebra over $k$. The canonical involution of $\mathcal{B}$ is denoted by $*$. For a $k$-algebra $R$, we put $\mathcal{B}_{R}=B \otimes R$. Let $n$ be a positive integer. Let $\mathrm{M}_{n}(\mathcal{B})$ be the set of $i$ by $i$ matrices with entries in $\mathcal{B}$, which is an associative $k$-algebra. We denote by $\mathcal{T}$ and $\mathcal{N}$ the reduced trace and the reduced norm of the simple algebra $\mathrm{M}_{n}(\mathcal{B})$. It is known that $x \in \mathrm{M}_{n}(\mathcal{B})$ is invertible if and only if $\mathcal{N}(x)$ is invertible. The group of invertible elements is denoted by $\mathrm{GL}_{n}(\mathcal{B})$. For $x=\left(x_{i j}\right) \in \mathrm{M}_{n}(\mathcal{B})$, we define $x^{\iota}=\left(x_{j i}^{*}\right) \in \mathrm{M}_{n}(\mathcal{B})$. Then $\iota$ is an involution of the algebra $\mathrm{M}_{n}(\mathcal{B})$. Let $\mathrm{H}_{n}(\mathcal{B})=\left\{x \in \mathrm{M}_{n}(\mathcal{B}) \mid x^{\iota}=x\right\}$, the space of Hermitian forms of degree $n$. Then $\mathrm{GL}_{n}(\mathcal{B})$ acts on this space via

$$
(g, x) \longmapsto g \cdot x=g x g^{\iota}, \quad g \in \mathrm{GL}_{n}(\mathcal{B}), x \in \mathrm{H}_{n}(\mathcal{B}),
$$

which is a linear representation of $\mathrm{GL}_{n}(\mathcal{B})$. It is known that there is a polynomial of degree $n$ on $\mathrm{H}_{n}(\mathcal{B})$ which is a square root of $\mathcal{N}$. This is uniquely determined if we assume the value at $1_{n} \in \mathrm{H}_{n}(\mathcal{B})$ is 1 . We denote this polynomial by Pfaff $_{n}$, since this coincides with the classical Pfaffian when $\mathcal{B} \cong \mathrm{M}_{2}(k)$. By definition,

$$
\operatorname{Pfaff}_{n}\left(1_{n}\right)=1, \quad \operatorname{Pfaff}_{n}(x)^{2}=\mathcal{N}(x), \quad \operatorname{Pfaff}_{n}(g \cdot x)=\mathcal{N}(g) \operatorname{Pfaff}_{n}(x)
$$

for $g \in \mathrm{GL}_{n}(\mathcal{B}), x \in \mathrm{H}_{n}(\mathcal{B})$.

What we are in interest is the representation

$$
G=\mathrm{GL}_{n}(\mathcal{B}) \times \mathrm{GL}_{2}, \quad V=\mathrm{H}_{n}(\mathcal{B}) \otimes k^{2} \quad(n=2,3),
$$

where we consider the outer tensor product representation. The representation $(G, V)$ becomes a prehomogeneous vector space if and only if $n=1,2,3$. Hence we consider the case $n=2,3$ 
for the rest of this paper (the case $n=1$ is not interesting from our view in this paper.) We regard this as a representation of algebraic group over $k$. This is an inner form of

$$
\left(\mathrm{GL}_{2 n} \times \mathrm{GL}_{2}, \wedge^{2} k^{2 n} \otimes k^{2}\right)
$$

and in the case $\mathcal{B} \cong \mathrm{M}_{2}(k)$ they are equivalent. We say the representation (3.1) split if $\mathcal{B} \cong \mathrm{M}_{2}(k)$ and non-split otherwise. It is well known that $\mathcal{B} \otimes k^{\mathrm{s}} \cong \mathrm{M}_{2}\left(k^{\mathrm{s}}\right)$. Hence we have:

Proposition 3.1. The representation (3.1) is equivalent to (3.2) over $k^{\mathrm{s}}$.

We describe the action more explicitly. Throughout this paper, we express elements of $G$ as $g=\left(g_{1}, g_{2}\right)$ where $g_{1} \in \mathrm{GL}_{n}(\mathcal{B}), g_{2} \in \mathrm{GL}_{2}$ and $V \cong \mathrm{H}_{n}(\mathcal{B}) \oplus \mathrm{H}_{n}(\mathcal{B})$ as $x=\left(x_{1}, x_{2}\right)$ where $x_{i} \in \mathrm{H}_{n}(\mathcal{B})$. We call $V$ the space of pairs of binary (resp. ternary) Hermitian forms if $n=2$ (resp. $n=3$ ). We identify $x=\left(x_{1}, x_{2}\right) \in V$ with the Hermition form $x(v)=x_{1} v_{1}+x_{2} v_{2}$ of linear form in the variables $v_{1}$ and $v_{2}$, which we collect into the row vector $v=\left(v_{1}, v_{2}\right)$. Then the left action of $g \in G$ on $x \in V$ is defined by

$$
(g \cdot x)(v)=g_{1} \cdot x\left(v g_{2}\right)=g_{1} x\left(v g_{2}\right) g_{1}^{\iota},
$$

or, in other words,

$$
\left(g_{1},\left(\begin{array}{ll}
a & b \\
c & d
\end{array}\right)\right) \cdot\left(x_{1}, x_{2}\right)=\left(g_{1}\left(a x_{1}+b x_{2}\right) g_{1}^{\iota}, g_{1}\left(c x_{1}+d x_{2}\right) g_{1}^{\iota}\right)
$$

We put $F_{x}(v)=\operatorname{Pfaff}_{n}(x(v))$. This is a binary quadratic form (resp. cubic form) in variables $v=\left(v_{1}, v_{2}\right)$ if $n=2$ (resp. $n=3$ ), and the discriminant $P(x)(x \in V)$ is a polynomial in $V$. The polynomial $P(x)$ is characterized by

$$
P(x)=\prod_{1 \leq i<j \leq n}\left(\alpha_{i} \beta_{j}-\alpha_{j} \beta_{i}\right)^{2} \quad \text { for } \quad F_{x}(v)=\prod_{1 \leq i \leq n}\left(\alpha_{i} v_{1}-\beta_{i} v_{2}\right), \quad x \in V\left(k^{\mathrm{s}}\right) .
$$

We define $\chi(g)=\mathcal{N}\left(g_{1}\right) \operatorname{det}\left(g_{2}\right)$ for $n=2$ and $\chi(g)=\mathcal{N}\left(g_{1}\right)^{2} \operatorname{det}\left(g_{2}\right)^{3}$ for $n=3$. Then one can easily see that

$$
P(g x)=\chi(g)^{2} P(x)
$$

and hence $P(x)$ is a relative invariant polynomial with respect to the character $\chi^{2}$. Let $S=$ $\{x \in V \mid P(x)=0\}$ and $V^{\mathrm{ss}}=\{x \in V \mid P(x) \neq 0\}$ and call them the set of unstable points and semi-stable points, respectively. That is, $x \in V$ is semi-stable if and only if $F_{x}(v)$ does not have a multiple root in $\mathbb{P}^{1}=\left\{\left(v_{1}: v_{2}\right)\right\}$.

We now consider rational points of $V^{\text {ss }}$ and its stabilizer structure.

Definition 3.2. (1) Let $\mathcal{P}_{2}$ (resp. $\mathcal{P}_{3}$ ) be the set of equivalence classes of $k$-forms of the variety $\operatorname{Proj} k\left[v_{1}, v_{2}\right] /\left(v_{1} v_{2}\right)$ (resp. Proj $k\left[v_{1}, v_{2}\right] /\left(v_{1} v_{2}\left(v_{1}-v_{2}\right)\right)$.

(2) For $n=2,3$, let $\mathcal{A}_{n}$ be set of isomorphism classes of separable $k$-algebras of dimension $n$.

We note that the contravariant functor

$$
s: \mathcal{P}_{n} \longrightarrow \mathcal{A}_{n}, \quad Z \longmapsto s(Z)=\Gamma\left(Z, \mathcal{O}_{Z}\right)
$$

gives a categorical equivalence between $\mathcal{P}_{n}$ and $\mathcal{A}_{n}$.

Definition 3.3. For $x \in V^{\mathrm{ss}}(k)$, we define

$$
\begin{aligned}
& Z_{x}=\operatorname{Proj} k\left[v_{1}, v_{2}\right] /\left(F_{x}(v)\right), \\
& L_{x}=\Gamma\left(Z_{x}, \mathcal{O}_{Z_{x}}\right) .
\end{aligned}
$$

Since $V^{\mathrm{ss}}(k)$ is the set of $x$ such that $F_{x}$ does not have a multiple root, $Z_{x} \in \mathcal{P}_{n}$ is a variety over $k$ and $L_{x} \in \mathcal{A}_{n}$. By definition $Z_{x}$ depends only on the $G(k)$-orbit of $x$ and hence we have a map $G(k) \backslash V^{\mathrm{ss}}(k) \rightarrow \mathcal{P}_{n}$. To describe the fiber structure, Wright and Yukie [WY92] interpreted this map in terms of Galois cohomology. We give a refined version of their argument. 
We fix an arbitrary element $w \in V^{\mathrm{ss}}(k)$. By definition, $\mathcal{P}_{n}$ is the set of equivalence classes of $k$-forms of $Z_{w}$. Hence by Theorem 2.6 there is the canonical bijection

$$
d_{w}: \mathrm{H}^{1}\left(k, \operatorname{Aut}\left(Z_{w}\right)\right) \longrightarrow \mathcal{P}_{n} .
$$

Let $G_{w}=\{g \in G \mid g \cdot w=w\}$ be the stabilizer and $G_{w}^{\circ}$ its identity component. If $g=\left(g_{1}, g_{2}\right) \in G_{w}$, then $\mathcal{N}\left(g_{1}\right) F_{w}\left(v g_{2}\right)=F_{w}(v)$. Hence each element of $G_{w}$ defines an automorphism of $Z_{w}$ and the map

$$
c_{w}: G_{w} \longrightarrow \operatorname{Aut}\left(Z_{w}\right), \quad g=\left(g_{1}, g_{2}\right) \longmapsto\left(Z_{w} \ni v \longmapsto v g_{2} \in Z_{w}\right)
$$

gives a homomorphism of algebraic groups. We note that we assume Aut $\left(Z_{w}\right)$ acts on $Z_{w}$ from the right.

Let us consider the following condition:

Condition 3.4. (1) For any $w \in V^{\mathrm{ss}}(k)$, the map $g \mapsto g w$ gives an isomorphism $G / G_{w} \rightarrow V^{\text {ss }}$ as varieties over $k$. We denote by $a_{w}$ the inverse map;

$$
a_{w}: V^{\mathrm{ss}} \longrightarrow G / G_{w} \text {. }
$$

(2) The canonical injection $G\left(k^{\mathrm{s}}\right) / G_{w}\left(k^{\mathrm{s}}\right) \rightarrow\left(G / G_{w}\right)\left(k^{\mathrm{s}}\right)$ is bijective.

(3) For any $w \in V^{\mathrm{ss}}(k)$, the sequence

$$
1 \longrightarrow G_{w}^{\circ} \longrightarrow G_{w} \stackrel{c_{w}}{\longrightarrow} \operatorname{Aut}\left(Z_{w}\right) \longrightarrow 1
$$

is exact.

(4) We have $\mathrm{H}^{1}(k, G)=\{1\}$.

Proposition 3.5. For the prehomogeneous vector space (3.1), Condition 3.4 hold.

Proof. (1) and (2) are proved by Yukie [Y97a] for a general irreducible regular prehomogeneous vector space. For (3) we may assume $k=k^{\mathrm{s}}$. Then $(G, V)$ is the split form. Since the exactness is proved in [WY92] for one element, the same is true for any its $G(k)$-orbit. By $(2) V^{\mathrm{ss}}(k)$ is a single $G(k)$-orbit and we have (3). (4) is a consequence of Proposition $2.7(2)$.

From (2) and (4) of Condition 3.4, we have the following by Theorem 2.3.

Proposition 3.6. The canonical map

$$
b_{w}: G(k) \backslash\left(G / G_{w}\right)(k) \longrightarrow \mathrm{H}^{1}\left(k, G_{w}\right)
$$

is bijective.

Definition 3.7. The map $a_{w}$ induces a bijective map $G(k) \backslash V^{\mathrm{ss}}(k) \rightarrow G(k) \backslash\left(G / G_{w}\right)(k)$, which we also denote by $a_{w}$. Also the map $\mathrm{H}^{1}\left(k, G_{w}\right) \rightarrow \mathrm{H}^{1}\left(k\right.$, Aut $\left.\left(Z_{w}\right)\right)$ induced by $c_{w}$ is also denoted by $c_{w}$. We define $\Phi_{w}$ by the following composition;

$$
\Phi_{w}=d_{w} \circ c_{w} \circ b_{w} \circ a_{w}: \quad G(k) \backslash V^{\mathrm{ss}}(k) \longrightarrow \mathcal{P}_{n} .
$$

Theorem 3.8. For $x \in V^{\mathrm{ss}}(k)$, we have $\Phi_{w}(G(k) \cdot x)=Z_{x}$. Especially $\Phi_{w}$ does not depends on the choice of $w \in V^{\mathrm{ss}}(k)$.

Proof. By (1) and (2) of Condition 3.4, $V^{\mathrm{ss}}\left(k^{\mathrm{s}}\right)$ is a single $G\left(k^{\mathrm{s}}\right)$-orbit. Take $g \in G\left(k^{\mathrm{s}}\right)$ such that

$$
x=g \cdot w, \quad g=\left(g_{1}, g_{2}\right) .
$$

Then $b_{w}\left(a_{w}(x)\right) \in \mathrm{H}^{1}\left(k, G_{w}\right)$ is the class of the 1-cocycle $\left(g^{-1} g^{\sigma}\right)_{\sigma \in \Gamma}$. Hence $c_{w}\left(b_{w}\left(a_{w}(y)\right)\right) \in$ $\mathrm{H}^{1}\left(k\right.$, Aut $\left.\left(Z_{w}\right)\right)$ is the class of the 1-cocycle $\left(c_{w}\left(g^{-1} g^{\sigma}\right)\right)_{\sigma \in \Gamma}$.

On the other side since $x=g \cdot w, F_{x}(v)$ coincides with $F_{w}\left(v g_{2}\right)$ up to non-zero constant. Hence

$$
\psi: Z_{x} \times_{k} k^{\mathrm{s}} \longrightarrow Z_{w} \times_{k} k^{\mathrm{s}}, \quad v \longmapsto \psi(v)=v g_{2}
$$


is an isomorphism of varieties over $k^{\mathrm{s}}$. Let

$$
h_{Z_{x}}(\sigma)=\sigma_{Z_{w}} \circ \psi \circ \sigma_{Z_{x}}^{-1} \circ \psi^{-1} \in \operatorname{Aut}_{k^{\mathrm{s}}}\left(Z_{w} \times_{k} k^{\mathrm{s}}\right) .
$$

Then $d_{w}^{-1}\left(Z_{x}\right)$ is the cohomology class of $\left(h_{Z_{x}}(\sigma)\right)_{\sigma \in \Gamma}$. Also for $v \in\left(Z_{w} \times_{k} k^{\mathrm{s}}\right) \subset \mathbb{P}^{1}$,

$$
\begin{aligned}
h_{Z_{x}}(\sigma)(v) & =\left(\sigma_{Z_{w}} \circ \psi \circ \sigma_{Z_{x}}^{-1}\right)\left(v g_{2}^{-1}\right)=\left(\sigma_{Z_{w}} \circ \psi\right)\left(v^{\sigma^{-1}}\left(g_{2}^{-1}\right)^{\sigma^{-1}}\right) \\
& =\sigma_{Z_{w}}\left(v^{\sigma^{-1}}\left(g_{2}^{-1}\right)^{\sigma^{-1}} g_{2}\right)=v g_{2}^{-1} g_{2}^{\sigma},
\end{aligned}
$$

which shows that $h_{Z_{x}}(\sigma)=c_{w}\left(g^{-1} g^{\sigma}\right)$. Hence we have $\left(c_{w} \circ b_{w} \circ a_{w}\right)(x)=d_{w}^{-1}\left(Z_{x}\right)$ and this finishes the proof.

Since $\Phi_{w}$ does not depend on $w$, we drop the subscript $w$ and write $\Phi$ from now on. As a corollary to the theorem above, we obtain the following;

Corollary 3.9. For $x \in V^{\mathrm{ss}}(k)$, the fiber $\Phi^{-1}\left(Z_{x}\right)$ is parameterized by the set:

$$
\mathrm{H}^{1}\left(k, G_{x}^{\circ}\right) / \operatorname{Aut}\left(Z_{x}\right)(k),
$$

where the action in the right hand side is determined by the exact sequence in Condition 3.4 (3). Moreover, if a cohomology class in $\mathrm{H}^{1}\left(k, G_{x}^{\circ}\right)$ is represented by 1-cocycle $\left(g^{-1} g^{\sigma}\right)_{\sigma \in \Gamma}$ for some $g \in G\left(k^{\mathrm{s}}\right)$, then the corresponding element in $\Phi^{-1}\left(Z_{x}\right)$ is the orbit of $g x \in V^{\mathrm{ss}}(k)$.

Proof. We regard $\Phi$ as the composition $d_{x} \circ c_{x} \circ b_{x} \circ a_{x}$. Since $d_{x}, b_{x}$ and $a_{x}$ are bijective, we consider the fiber of $c_{x}: \mathrm{H}^{1}\left(k, G_{x}\right) \rightarrow \mathrm{H}^{1}\left(k\right.$, Aut $\left.\left(Z_{x}\right)\right)$. Since $d_{x}^{-1}\left(Z_{x}\right) \in \mathrm{H}^{1}\left(k\right.$, Aut $\left.\left(Z_{x}\right)\right)$ is the trivial class, the first statement follows from the exactness of the sequence

$$
\mathrm{H}^{1}\left(k, G_{x}^{\circ}\right) \longrightarrow \mathrm{H}^{1}\left(k, G_{x}\right) \stackrel{c_{x}}{\longrightarrow} \mathrm{H}^{1}\left(k, \operatorname{Aut}\left(Z_{x}\right)\right)
$$

and the injectivity of the map $\mathrm{H}^{1}\left(k, G_{x}^{\circ}\right) / \operatorname{Aut}\left(Z_{x}\right)(k) \rightarrow \mathrm{H}^{1}\left(k, G_{x}\right)$ those asserted in Theorem 2.2. The second statement follows from the definitions of maps of the Galois cohomology.

Remark 3.10. We prove results above using Condition 3.4 and formulae (3.4), (3.8), (3.9). It is written explicitly or implicitly in [WY92, KY97] that all the prehomogeneous vector spaces they considered satisfy the same condition for certain $Z_{x}$ defined geometrically, hence our arguments are applicable for their cases. In [WY92, KY97] they chose a specific element $w$ so that the exact sequence of 3.4 (3) splits. Our approach does not need to choose such an element, and hence fitting in cases that there does not exist $w$ such that $c_{w}$ splits as we treated in [T04].

Definition 3.11. We put $\Psi=s \circ \Phi: G(k) \backslash V^{\mathrm{ss}}(k) \rightarrow \mathcal{A}_{n}$.

For $k$-algebra $R$, the norm map $\mathcal{B}_{R} \rightarrow R$ induced from $\mathcal{N}: \mathcal{B} \rightarrow k$ is also denoted by $\mathcal{N}$. We often regard $\mathcal{B}^{\times}$as an algebraic group over $k$. We hope the meaning is clear from the context.

3.2. The case of $D_{5}$ type. We now consider the case $n=2$ and $n=3$ separately. In this subsection we consider the case $n=2$, i.e., the space of pairs of binary Hermitian forms. Let

$$
w=\left(\left(\begin{array}{ll}
0 & 0 \\
0 & 1
\end{array}\right),\left(\begin{array}{ll}
1 & 0 \\
0 & 0
\end{array}\right)\right) \in V(k) .
$$

Proposition 3.12. We have $w \in V^{\mathrm{ss}}(k), L_{w}=k \times k$ and

$$
G_{w}^{\circ}=\left\{\left(\left(\begin{array}{cc}
b_{1} & 0 \\
0 & b_{2}
\end{array}\right),\left(\begin{array}{cc}
t_{1} & 0 \\
0 & t_{2}
\end{array}\right)\right) \mid \begin{array}{l}
b_{i} \in \mathcal{B}^{\times}, t_{i} \in \mathbb{G}_{m}, \\
t_{1} \mathcal{N}\left(b_{1}\right)=t_{2} \mathcal{N}\left(b_{2}\right)=1
\end{array}\right\} .
$$

Proof. Since $F_{w}(v)=v_{1} v_{2}$, we have $w \in V^{\mathrm{ss}}(k)$ and $L_{w}=k \times k$. Let $H$ denote the group of the right hand side. We immediately see that elements of $H$ stabilize $w$ and that $H$ is connected. Hence $H \subset G_{w}^{\circ}$. Since

$$
\operatorname{dim} H=8=\operatorname{dim} G-\operatorname{dim} V=\operatorname{dim} G-\operatorname{dim}\left(G / G_{w}\right)=\operatorname{dim} G_{w}=\operatorname{dim} G_{w}^{\circ}
$$

and both $H$ and $G_{w}^{\circ}$ are connected, they coincide. 
Let $L=k(\alpha)$ is a quadratic field extension. Let $\nu$ be the generator of $\operatorname{Gal}(L / k)$. Put $a_{1}=\alpha+\alpha^{\nu}, a_{2}=\alpha \alpha^{\nu}$ and

$$
x_{\alpha}=\left(\left(\begin{array}{cc}
0 & 1 \\
1 & a_{1}
\end{array}\right),\left(\begin{array}{cc}
1 & a_{1} \\
a_{1} & a_{1}^{2}-2 a_{2}
\end{array}\right)\right) \in V(k) .
$$

Let

$$
g_{\alpha}=\left(\left(\begin{array}{cc}
1 & 1 \\
\alpha & \alpha^{\nu}
\end{array}\right), \frac{1}{\alpha-\alpha^{\nu}}\left(\begin{array}{cc}
-1 & 1 \\
-\alpha^{\nu} & \alpha
\end{array}\right)\right) \in G(L)
$$

By computation we have $x_{\alpha}=g_{\alpha} w$.

Proposition 3.13. We have $x_{\alpha} \in V^{\mathrm{ss}}(k), L_{x_{\alpha}}=L$, and $G_{x_{\alpha}}^{\circ} \cong \mathcal{R}_{L / k}\left(\mathcal{B}_{L}^{\times}\right)$.

Proof. Since $F_{x_{\alpha}}(v)=-\left(v_{1}^{2}+a_{1} v_{1} v_{2}+a_{2} v_{2}^{2}\right)=-\left(v_{1}+\alpha v_{2}\right)\left(v_{1}+\alpha^{\nu} v_{2}\right)$, we have $x_{\alpha} \in V^{\mathrm{ss}}(k)$ and $L_{x_{\alpha}}=L$. We consider $G_{x}^{\circ}$. Let $R$ be any $k$-algebra. Then since $x=g_{\alpha} w, G_{x}^{\circ}(R \otimes L)=$ $g_{\alpha} G_{w}^{\circ}(R \otimes L) g_{\alpha}^{-1}$. Also $G_{x}^{\circ}(R)=\left\{p \in G_{x}^{\circ}(R \otimes L) \mid p^{\nu}=p\right\}$. Using $g_{\alpha}^{\nu}=g_{\alpha} \tilde{\nu}$ where

$$
\tilde{\nu}=\left(\left(\begin{array}{ll}
0 & 1 \\
1 & 0
\end{array}\right),\left(\begin{array}{ll}
0 & 1 \\
1 & 0
\end{array}\right)\right)
$$

by computation we have

$$
G_{x}^{\circ}(R)=g_{\alpha}\left\{\left(\left(\begin{array}{cc}
b & 0 \\
0 & b^{\nu}
\end{array}\right),\left(\begin{array}{cc}
t & 0 \\
0 & t^{\nu}
\end{array}\right)\right) \mid \begin{array}{l}
b \in\left(\mathcal{B}_{L} \otimes R\right)^{\times} \\
t \in(L \otimes R)^{\times}, t \mathcal{N}(b)=1
\end{array}\right\} g_{\alpha}^{-1} .
$$

Hence $G_{x}^{\circ}(R) \cong\left(\mathcal{B}_{L} \otimes R\right)^{\times}$and this isomorphism is functorial with respect to $k$-algebras. This proves the proposition.

Proposition 3.14. The map $\Psi: G(k) \backslash V^{\mathrm{ss}}(k) \rightarrow \mathcal{A}_{2}$ is bijective.

Proof. By Propositions 3.12 and 3.13, $\Psi$ is surjective. By Proposition $2.7, \mathrm{H}^{1}\left(k, \mathcal{B}^{\times} \times \mathcal{B}^{\times}\right)=$ $\mathrm{H}^{1}\left(k, \mathcal{R}_{L / k}\left(\mathcal{B}_{L}^{\times}\right)\right)=\{1\}$ where $L / k$ is a quadratic field extension. This combined with Propositions 3.12 and 3.13 shows that for any $L \in \mathcal{A}_{2}$, there exists $x \in V^{\mathrm{ss}}(k)$ such that $L_{x}=L$ and $\mathrm{H}^{1}\left(k, G_{x}^{\circ}\right)=\{1\}$. Hence by Corollary 3.9 each fiber of elements of $\mathcal{A}_{2}$ consists of a single orbit. Hence $\Psi$ is injective.

We summarize the result of this subsection as follows.

Theorem 3.15. Let $n=2$.

(1) The map

$$
\Psi: G(k) \backslash V^{\mathrm{ss}}(k) \longrightarrow \mathcal{A}_{2}, \quad G(k) \cdot x \longmapsto L_{x}
$$

is bijective.

(2) For $x \in V^{\mathrm{ss}}(k), G_{x}^{\circ} \cong \mathcal{R}_{L_{x} / k}\left(\mathcal{B}_{L_{x}}^{\times}\right)$.

3.3. The case of $E_{7}$ type: Statements. The case $n=3$ becomes a little more complicated than the case $n=2$, and we give statements and proofs separately. We give statements in this subsection and prove in the next subsection. For the space of pairs of ternary Hermitian forms, the following theorems hold.

Theorem 3.16. Let $n=3$.

(1) The map

$$
\Psi: G(k) \backslash V^{\mathrm{ss}}(k) \longrightarrow \mathcal{A}_{3}, \quad G(k) \cdot x \longmapsto L_{x}
$$

is surjective, and the fiber of any $L \in \mathcal{A}_{3}$ is parameterized by the set

$$
\left(k^{\times} \cdot \operatorname{Im}\left(\mathcal{N}: \mathcal{B}_{L}^{\times} \rightarrow L^{\times}\right)\right) \backslash L^{\times} / \operatorname{Aut}_{k}(L) .
$$

(2) Let $x \in V^{\mathrm{ss}}(k)$. There exists an exact sequence of algebraic groups

$$
1 \longrightarrow G_{x}^{\circ} \longrightarrow \mathbb{G}_{m} \times \mathcal{R}_{L_{x} / k}\left(\mathcal{B}_{L_{x}}^{\times}\right) \stackrel{\varphi_{x}}{\longrightarrow} \mathcal{R}_{L_{x} / k}\left(\mathbb{G}_{m}\right) \longrightarrow 1,
$$

where $\varphi_{x}$ is given by $\varphi_{x}(t, b)=t \mathcal{N}(b)$. 
A parameterization in Theorem 3.16 (1) is given by:

Theorem 3.17. (1) Let $L=k \times k \times k$. Then $\left(p_{1}, p_{2}, p_{3}\right) \in\left(k^{\times}\right)^{3}=L^{\times}$corresponds to the orbit of

$$
\left(\left(\begin{array}{ccc}
0 & 0 & 0 \\
0 & p_{2} & 0 \\
0 & 0 & -p_{3}
\end{array}\right),\left(\begin{array}{ccc}
p_{1} & 0 & 0 \\
0 & -p_{2} & 0 \\
0 & 0 & 0
\end{array}\right)\right)
$$

(2) Let $L=k \times F$ where $F=k(\alpha)$ is a quadratic field extension of $k$. Let $\nu$ be the generator of $\operatorname{Gal}(F / k)$. Then $(p, \lambda) \in k^{\times} \times F^{\times}=L^{\times}$corresponds to the orbit of

$$
\left(\left(\begin{array}{ccc}
0 & 0 & 0 \\
0 & \Lambda_{0} & \Lambda_{1} \\
0 & \Lambda_{1} & \Lambda_{2}
\end{array}\right),\left(\begin{array}{ccc}
-p & 0 & 0 \\
0 & \Lambda_{1} & \Lambda_{2} \\
0 & \Lambda_{2} & \Lambda_{3}
\end{array}\right)\right)
$$

where we put $\Lambda_{i}=\left(\lambda \alpha^{i}-\left(\lambda \alpha^{i}\right)^{\nu}\right) /\left(\alpha-\alpha^{\nu}\right) \in k$.

(3) Let $L=k(\beta)$ be a cubic field extension of $k$. We denote by $L^{\text {nc }}$ the normal closure of $L$, and $\beta_{1}=\beta, \beta_{2}=\beta^{\tau}, \beta_{3}=\beta^{\mu}\left(\tau, \mu \in \operatorname{Gal}\left(L^{\mathrm{nc}} / k\right)\right)$ the all conjugates of $\beta$. Then $\delta \in E^{\times}$ corresponds to the orbit of

$$
\left(\left(\begin{array}{ccc}
\Delta_{0} & \Delta_{1} & \Delta_{2} \\
\Delta_{1} & \Delta_{2} & \Delta_{3} \\
\Delta_{2} & \Delta_{3} & \Delta_{4}
\end{array}\right),\left(\begin{array}{ccc}
\Delta_{1} & \Delta_{2} & \Delta_{3} \\
\Delta_{2} & \Delta_{3} & \Delta_{4} \\
\Delta_{3} & \Delta_{4} & \Delta_{5}
\end{array}\right)\right)
$$

where we put $\delta_{1}=\delta, \delta_{2}=\delta^{\tau}, \delta_{3}=\delta^{\mu}$ and

$$
\Delta_{i}=\frac{\delta_{1} \beta_{1}^{i}\left(\beta_{2}-\beta_{3}\right)+\delta_{2} \beta_{2}^{i}\left(\beta_{3}-\beta_{1}\right)+\delta_{3} \beta_{3}^{i}\left(\beta_{1}-\beta_{2}\right)}{\left(\beta_{1}-\beta_{2}\right)\left(\beta_{2}-\beta_{3}\right)\left(\beta_{3}-\beta_{1}\right)} \in k .
$$

3.4. The case of $E_{7}$ type: Proofs. We now give proofs of Theorems 3.16 and 3.17 in order. We keep the notation in the previous subsection. As for the $D_{5}$ case, we first choose a specific element in the fiber for each $L \in \mathcal{A}_{3}$ and determine its stabilizer structure.

We write "diagonal" elements of $G$ as

$$
\mathrm{d}\left(b_{1}, b_{2}, b_{3}, t\right)=\left(\left(\begin{array}{ccc}
b_{1} & 0 & 0 \\
0 & b_{2} & 0 \\
0 & 0 & b_{3}
\end{array}\right),\left(\begin{array}{cc}
t & 0 \\
0 & t
\end{array}\right)\right) \in G \quad\left(b_{i} \in \mathcal{B}^{\times}, t \in \mathbb{G}_{m} .\right)
$$

Let

$$
w=\left(\left(\begin{array}{ccc}
0 & 0 & 0 \\
0 & 1 & 0 \\
0 & 0 & -1
\end{array}\right),\left(\begin{array}{ccc}
1 & 0 & 0 \\
0 & -1 & 0 \\
0 & 0 & 0
\end{array}\right)\right) \in V(k)
$$

We define

$$
K^{w}=\left\{\mathrm{d}\left(b_{1}, b_{2}, b_{3}, t\right) \mid b_{i} \in \mathcal{B}^{\times}, t \in \mathbb{G}_{m}\right\} \subset G
$$

and

$$
\varphi_{w}: K^{w} \longrightarrow \mathbb{G}_{m}^{3}, \quad \mathrm{~d}\left(b_{1}, b_{2}, b_{3}, t\right) \longmapsto\left(t \mathcal{N}\left(b_{1}\right), t \mathcal{N}\left(b_{2}\right), t \mathcal{N}\left(b_{3}\right)\right) .
$$

Then the following can be proved similarly to Proposition 3.12.

Proposition 3.18. We have $w \in V^{\mathrm{ss}}(k), L_{w}=k \times k \times k$ and $G_{w}^{\circ}=\operatorname{ker}\left(\varphi_{w}\right)$.

Let $L=k \times F \in \mathcal{A}_{3}$ where $F=k(\alpha) / k$ is a quadratic field extension. Put $a_{1}=\alpha+\alpha^{\nu}$, $a_{2}=\alpha \alpha^{\nu}$ and

$$
x_{\alpha}=\left(\left(\begin{array}{ccc}
0 & 0 & 0 \\
0 & 0 & 1 \\
0 & 1 & a_{1}
\end{array}\right),\left(\begin{array}{ccc}
-1 & 0 & 0 \\
0 & 1 & a_{1} \\
0 & a_{1} & a_{1}^{2}-a_{2}
\end{array}\right)\right) \in V(k) .
$$


Let

$$
g_{\alpha}=\left(\left(\begin{array}{ccc}
1 & 0 & 0 \\
0 & 1 & 1 \\
0 & \alpha & \alpha^{\nu}
\end{array}\right), \frac{1}{\alpha-\alpha^{\nu}}\left(\begin{array}{cc}
1 & 0 \\
\alpha^{\nu} & \alpha^{\nu}-\alpha
\end{array}\right)\right) \in G(F) .
$$

By computation we have $x_{\alpha}=g_{\alpha} w$. Let

$$
K^{x_{\alpha}}(k)=g_{\alpha}\left\{\mathrm{d}\left(b_{1}, b_{2}, b_{2}^{\nu}, t\right) \mid b_{1} \in \mathcal{B}^{\times}, b_{2} \in \mathcal{B}_{F}^{\times}, t \in k^{\times}\right\} g_{\alpha}^{-1} .
$$

Lemma 3.19. We have $K^{x_{\alpha}}(k) \subset G(k)$.

Proof. Let $p=g_{\alpha} \mathrm{d}\left(b_{1}, b_{2}, b_{2}^{\nu}, t\right) g_{\alpha}^{-1} \in K^{x_{\alpha}}(k)$. We already know $p \in G(F)$. Since $g_{\alpha}^{\nu}=g_{\alpha} \widetilde{\nu}$ where

$$
\widetilde{\nu}=\left(\left(\begin{array}{lll}
1 & 0 & 0 \\
0 & 0 & 1 \\
0 & 1 & 0
\end{array}\right),\left(\begin{array}{cc}
-1 & 0 \\
1 & 1
\end{array}\right)\right)
$$

we have $p^{\nu}=g_{\alpha} \widetilde{\nu} \mathrm{d}\left(b_{1}, b_{2}^{\nu}, b_{2}, t\right) \widetilde{\nu}^{-1} g_{\alpha}^{-1}=g_{\alpha} \mathrm{d}\left(b_{1}, b_{2}, b_{2}^{\nu}, t\right) g_{\alpha}^{-1}=p$. Hence $p \in G(k)$.

We regard $K^{x_{\alpha}}(k)$ as $k$-rational points of the algebraic group $K^{x_{\alpha}} \subset G$ over $k$ in an obvious manner. That is, for any $k$-algebra $R$,

$$
K^{x_{\alpha}}(R)=g_{\alpha}\left\{\mathrm{d}\left(b_{1}, b_{2}, b_{2}^{\nu}, t\right) \mid b_{1} \in \mathcal{B}_{R}^{\times}, b_{2} \in \mathcal{B}_{F \otimes R}^{\times}, t \in R^{\times}\right\} g_{\alpha}^{-1},
$$

which we can prove is a subgroup of $G(R)$ similarly to the lemma above. We define

$$
\varphi_{x_{\alpha}}: K^{x_{\alpha}} \longrightarrow \mathbb{G}_{m} \times \mathcal{R}_{F / k}\left(\mathbb{G}_{m}\right), \quad g_{\alpha} \mathrm{d}\left(b_{1}, b_{2}, b_{2}^{\nu}, t\right) g_{\alpha}^{-1} \longmapsto\left(t \mathcal{N}\left(b_{1}\right), t \mathcal{N}\left(b_{2}\right)\right) .
$$

Proposition 3.20. We have $x_{\alpha} \in V^{\mathrm{ss}}(k), L_{x_{\alpha}}=L$ and $G_{x_{\alpha}}^{\circ}=\operatorname{ker}\left(\varphi_{x_{\alpha}}\right)$.

Proof. Since

$$
F_{x_{\alpha}}(v)=v_{2}\left(v_{1}^{2}+a_{1} v_{1} v_{2}+a_{2} v_{2}^{2}\right)=v_{2}\left(v_{1}+\alpha v_{2}\right)\left(v_{1}+\alpha^{\nu} v_{2}\right),
$$

we have $x_{\alpha} \in V^{\mathrm{ss}}(k), L_{x_{\alpha}}=L$. Let $R$ be a $k$-algebra. We consider $G_{x_{\alpha}}^{\circ}(R)$. Since $x_{\alpha}=g_{\alpha} w$, $G_{x_{\alpha}}^{\circ}(R \otimes F)=g_{\alpha} G_{w}^{\circ}(R \otimes F) g_{\alpha}^{-1}$. Let

$$
p=g_{\alpha} \mathrm{d}\left(b_{1}, b_{2}, b_{3}, t\right) g_{\alpha}^{-1} \in G_{x_{\alpha}}^{\circ}(R \otimes F), \quad b_{i} \in\left(\mathcal{B}_{F} \otimes R\right)^{\times}, t \in(F \otimes R)^{\times}, t \mathcal{N}\left(b_{i}\right)=1 .
$$

Then $p=p^{\nu}$ if and only if $b_{1}=b_{1}^{\nu}, b_{3}=b_{2}^{\nu}$, and $t=t^{\nu}$. This shows $G_{x_{\alpha}}^{\circ}(R)=\operatorname{ker}\left(\varphi_{x_{\alpha}}\right)(R)$.

Let $L=k(\beta) / k$ be a cubic field extension. Put $b_{1}=\beta_{1}+\beta_{2}+\beta_{3}, b_{2}=\beta_{2} \beta_{3}+\beta_{3} \beta_{1}+\beta_{1} \beta_{2}$, $b_{3}=\beta_{1} \beta_{2} \beta_{3}, D=\left(\beta_{1}-\beta_{2}\right)\left(\beta_{2}-\beta_{3}\right)\left(\beta_{3}-\beta_{1}\right)$ and

$$
x_{\beta}=\left(\left(\begin{array}{ccc}
0 & 0 & 1 \\
0 & 1 & b_{1} \\
1 & b_{1} & b_{1}^{2}-b_{2}
\end{array}\right),\left(\begin{array}{ccc}
0 & 1 & b_{1} \\
1 & b_{1} & b_{1}^{2}-b_{2} \\
b_{1} & b_{1}^{2}-b_{2} & b_{1}^{3}-2 b_{1} b_{2}+b_{3}
\end{array}\right)\right) \in V(k) .
$$

Let

$$
g_{\beta}=\left(\left(\begin{array}{ccc}
1 & 1 & 1 \\
\beta_{1} & \beta_{2} & \beta_{3} \\
\beta_{1}^{2} & \beta_{2}^{2} & \beta_{3}^{2}
\end{array}\right), \frac{1}{D}\left(\begin{array}{cc}
\beta_{2}-\beta_{1} & \beta_{2}-\beta_{3} \\
\beta_{3}\left(\beta_{2}-\beta_{1}\right) & \beta_{1}\left(\beta_{2}-\beta_{3}\right)
\end{array}\right)\right) \in G\left(L^{\mathrm{nc}}\right) .
$$

By computation we have $x_{\beta}=g_{\beta} w$. Let

$$
K^{x_{\beta}}(k)=g_{\beta}\left\{\mathrm{d}\left(b, b^{\tau}, b^{\mu}, t\right) \mid b \in \mathcal{B}_{L}^{\times}, t \in k^{\times}\right\} g_{\beta}^{-1} .
$$

Lemma 3.21. We have $K^{x_{\beta}}(k) \subset G(k)$.

Proof. Let $L / k$ be a non-Galois cubic extension. By changing $\tau, \mu \in \operatorname{Gal}\left(L^{\mathrm{nc}} / k\right)$ if necessary, we assume they are of index 2 . Then since $\tau\left(\beta_{3}\right)=\beta_{3}$ and $\mu\left(\beta_{2}\right)=\beta_{2}$, we have $g_{\beta}^{\tau}=g_{\beta} \widetilde{\tau}$ and $g_{\beta}^{\mu}=g_{\beta} \widetilde{\mu}$ where

$$
\widetilde{\tau}=\left(\left(\begin{array}{lll}
0 & 1 & 0 \\
1 & 0 & 0 \\
0 & 0 & 1
\end{array}\right),\left(\begin{array}{cc}
1 & 1 \\
0 & -1
\end{array}\right)\right), \quad \widetilde{\mu}=\left(\left(\begin{array}{lll}
0 & 0 & 1 \\
0 & 1 & 0 \\
1 & 0 & 0
\end{array}\right),\left(\begin{array}{cc}
0 & -1 \\
-1 & 0
\end{array}\right)\right) .
$$


Also $\mu \tau \mu=\tau \mu \tau \in \operatorname{Gal}\left(L^{\mathrm{nc}} / k\right)$ fixes elements of $L$. Hence we can see any element $p=$ $g_{\beta} \mathrm{d}\left(b, b^{\tau}, b^{\mu}, t\right) g_{\beta}^{-1} \in G\left(L^{\mathrm{nc}}\right)$ is fixed by $\tau, \mu$, and consequently $p \in G(k)$. The case $L / k$ is Galois is similarly proved and we omit the repetition here.

As before we regard $K^{x_{\beta}}(k)$ as $k$-rational points of the algebraic group $K^{x_{\beta}} \subset G$ over $k$. We define

$$
\varphi_{x_{\beta}}: K^{x_{\beta}} \longrightarrow \mathcal{R}_{L / k}\left(\mathbb{G}_{m}\right), \quad g_{\beta} \mathrm{d}\left(b, b^{\tau}, b^{\mu}, t\right) g_{\beta}^{-1} \longmapsto t \mathcal{N}(b) .
$$

Proposition 3.22. We have $x_{\beta} \in V^{\mathrm{ss}}(k), L_{x_{\beta}}=L$ and $G_{x_{\beta}}^{\circ}=\operatorname{ker}\left(\varphi_{x_{\beta}}\right)$.

Proof. Since

$$
F_{x_{\beta}}(v)=-\left(v_{1}^{3}+b_{1} v_{1}^{2} v_{2}+b_{2} v_{1} v_{2}^{2}+b_{3} v_{2}^{3}\right)=-\left(v_{1}+\beta_{1} v_{2}\right)\left(v_{1}+\beta_{2} v_{2}\right)\left(v_{1}+\beta_{3} v_{2}\right),
$$

we have $x_{\beta} \in V^{\mathrm{ss}}(k), L_{x_{\beta}}=L$. We determine $G_{x_{\beta}}^{\circ}$. Consider the case $L / k$ a non-Galois cubic extension. We use the notation in the lemma above. Since $x_{\beta}=g_{\beta} w, G_{x_{\beta}}^{\circ}\left(L^{\mathrm{nc}}\right)=$ $g_{\beta} G_{w}^{\circ}\left(L^{\mathrm{nc}}\right) g_{\beta}^{-1}$. Let

$$
p=g_{\alpha} \mathrm{d}\left(b_{1}, b_{2}, b_{3}, t\right) g_{\alpha}^{-1} \in G_{x_{\alpha}}^{\circ}\left(L^{\mathrm{nc}}\right), \quad b_{i} \in \mathcal{B}_{L^{\mathrm{nc}}}^{\times}, t \in\left(L^{\mathrm{nc}}\right)^{\times}, t \mathcal{N}\left(b_{i}\right)=1 .
$$

Then $p=p^{\tau}=p^{\mu}$ if and only if $b_{2}=b_{1}^{\tau}, b_{3}=b_{1}^{\mu}, b_{1}=b_{1}^{\mu \tau \mu}=b_{1}^{\tau \mu \tau}$ and $t=t^{\tau}=t^{\mu}$. This shows $G_{x_{\beta}}^{\circ}(k)=\operatorname{ker}\left(\varphi_{x_{\beta}}\right)(k)$. The equality $G_{x_{\beta}}^{\circ}(R)=\operatorname{ker}\left(\varphi_{x_{\beta}}\right)(R)$ for a $k$-algebra $R$ is proved in the same way and thus we have $G_{x_{\beta}}^{\circ}=\operatorname{ker}\left(\varphi_{x_{\beta}}\right)$.

We now prove Theorems 3.16, 3.17 using Propositions 3.18, 3.20, 3.22 and Corollary 3.9.

Proof. We already know the map $\Psi$ is surjective and we consider the fiber structure. Let $x \in V^{\mathrm{ss}}(k)$ be either one of $w, x_{\alpha}$ or $x_{\beta}$ in (3.10), (3.11) or (3.12). For such $x$, we proved in Propositions 3.18, 3.20 and 3.22 that $G_{x}^{\circ}$ satisfies the exact sequence

$$
1 \longrightarrow G_{x}^{\circ} \longrightarrow K^{x} \stackrel{\varphi_{x}}{\longrightarrow} \mathcal{R}_{L_{x} / k}\left(\mathbb{G}_{m}\right) \longrightarrow 1
$$

where $K^{x} \cong \mathbb{G}_{m} \times \mathcal{R}_{L_{x} / k}\left(\mathcal{B}_{L_{x}}^{\times}\right)$. By Proposition $2.7, \mathrm{H}^{1}\left(k, K^{x}\right)=\{1\}$. Hence by Theorem 2.3 we have

$$
\mathrm{H}^{1}\left(k, G_{x}^{\circ}\right) \cong \varphi_{x}\left(K^{x}(k)\right) \backslash L_{x}^{\times} .
$$

Since $s$ is a contravariant functor,

$$
\operatorname{Aut}\left(Z_{x}\right)(k)=\operatorname{Aut}_{k}\left(Z_{x}\right) \longrightarrow \operatorname{Aut}_{k}\left(L_{x}\right), \quad \psi \longmapsto s^{*}\left(\psi^{-1}\right)
$$

gives the isomorphism of the groups. We later see their actions on respective sets are compatible. With the compatibility, by Corollary 3.9 we have the parameterization.

We give the explicit correspondence of this parameterization and determine the structure of stabilizers of the elements. First consider the case $x=w, L=L_{w}=k \times k \times k$. Take $p=\left(p_{1}, p_{2}, p_{3}\right) \in L^{\times}$. Let $g=\mathrm{d}\left(b_{1}, b_{2}, b_{3}, 1\right) \in K^{w}\left(k^{\mathrm{s}}\right)$ be in $\varphi_{w}^{-1}(p)$, i.e., $\mathcal{N}\left(b_{i}\right)=p_{i}$ for $i=1,2,3$. Then the class of $\left(g^{-1} g^{\sigma}\right)_{\sigma}$ corresponds to the class of $p$. Hence by Corollary 3.9, the class of $p \in L^{\times}$corresponds to $g w$, which equals to the element of Theorem 3.17 (1). Also by the form of $g w$ elements of $G_{w}^{\circ}$ stabilize $g w$. Comparing the dimensions, we have $G_{g w}^{\circ}=G_{w}^{\circ}$.

Next consider the case $x=x_{\beta}, L=L_{x}$ is a cubic Galois extension of $k$. Let $\theta \in \operatorname{Gal}\left(L_{x} / k\right)$ be the element satisfying $\beta_{2}=\beta_{1}^{\theta}, \beta_{3}=\beta_{2}^{\theta}$. Let $\delta \in L^{\times}$. We use the notation $\delta_{i}$ and $\Delta_{i}$ as in Theorem $3.17(3)$. Take $b \in\left(\mathcal{B}_{L} \otimes k^{\mathrm{s}}\right)^{\times}$such that $\mathcal{N}(b)=\delta$ and put $g=g_{\beta} \mathrm{d}\left(b, b^{\theta}, b^{\theta^{2}}, 1\right) g_{\beta}^{-1}$. Then $\varphi_{x}(g)=\delta$ and so the class of $\left(g^{-1} g^{\sigma}\right)_{\sigma}$ corresponds to the class of $\delta$. Hence by Corollary 3.9 , the class of $\delta \in L^{\times}$corresponds to $g x$. Since $x=g_{\beta} w$,

$$
g x=g_{\beta} \mathrm{d}\left(b, b^{\theta}, b^{\theta^{2}}, 1\right) w=g_{\beta}\left(\left(\begin{array}{ccc}
0 & 0 & 0 \\
0 & \delta^{\theta} & 0 \\
0 & 0 & -\delta^{\theta^{2}}
\end{array}\right),\left(\begin{array}{ccc}
\delta & 0 & 0 \\
0 & -\delta^{\theta} & 0 \\
0 & 0 & 0
\end{array}\right)\right)
$$


and thus equals to the element of Theorem 3.17 (3). Since elements of $G_{x}^{\circ}$ stabilize $g x$, we have $G_{g x}^{\circ}=G_{x}^{\circ}$. The remaining cases are proved similarly and we omit the details here.

We finally give the compatibility of actions of groups (3.14) on sets (3.13). Since the proofs are similar we consider the case $L=L_{x}$ is a cubic Galois extension of $k$. We use the notation above. We put

$$
\tilde{\theta}=\left(\left(\begin{array}{lll}
0 & 0 & 1 \\
1 & 0 & 0 \\
0 & 1 & 0
\end{array}\right),\left(\begin{array}{cc}
0 & 1 \\
-1 & -1
\end{array}\right)\right) \in G(k) .
$$

Then $g_{\beta}^{\theta}=g_{\beta} \tilde{\theta}$ and $\tilde{\theta} \in G_{w}$. Hence if we let $h=g_{\beta} \tilde{\theta} g_{\beta}^{-1}$, then we have $h \in G_{x}(k)$. Recall that we define $c_{x}: G_{x} \rightarrow \operatorname{Aut}\left(Z_{x}\right)$ in (3.4). By definition, $Z_{x}=\left\{\left(\beta_{1}:-1\right),\left(\beta_{2}:-1\right),\left(\beta_{3}:-1\right)\right\} \subset$ $\mathbb{P}^{1}$. We see by computation that $c_{x}(h): Z_{x} \rightarrow Z_{x}$ is given by

$$
\left(\beta_{1}:-1\right) \mapsto\left(\beta_{3}:-1\right), \quad\left(\beta_{2}:-1\right) \mapsto\left(\beta_{1}:-1\right), \quad\left(\beta_{3}:-1\right) \mapsto\left(\beta_{2}:-1\right) .
$$

Hence under the isomorphism in (3.14), $\theta \in \operatorname{Aut}\left(L_{x}\right)$ corresponds to $c_{x}(h) \in \operatorname{Aut}\left(Z_{x}\right)(k)$. By the definition of the action, $\left(g^{-1} g^{\sigma}\right)_{\sigma}^{c_{x}(h)}=\left(h^{-1} g^{-1} g^{\sigma} h^{\sigma}\right)_{\sigma}=\left(\left(h^{-1} g h\right)^{-1}\left(h^{-1} g h\right)^{\sigma}\right)_{\sigma}$. Since

$$
h^{-1} g h=g_{\beta} \tilde{\theta}^{-1} \mathrm{~d}\left(b, b^{\theta}, b^{\theta^{2}}, 1\right) \tilde{\theta} g_{\beta}^{-1}=g_{\beta} \mathrm{d}\left(b^{\theta}, b^{\theta^{2}}, b, 1\right) g_{\beta}^{-1}
$$

the image of $\delta^{\theta}$ under the bijection (3.13) is the cohomology class of the cocycle $\left(g^{-1} g^{\sigma}\right)_{\sigma}^{c_{x}(h)}$. This shows the compatibility of the actions.

3.5. Decomposition of the zeta function. We conclude this section with a brief discussion of the global zeta functions of the prehomogeneous vector space $(G, V)$. Let $k$ be a number field and $\mathbb{A}$ its adele ring. Let $(G, V)$ be either the $D_{5}$ or $E_{7}$ type. We put $\mathcal{A}_{2}^{\prime}=\left\{L \in \mathcal{A}_{2} \mid\right.$ $\left.\mathcal{A}_{2} \neq k \times k\right\}$ and $\mathcal{A}_{3}^{\prime}=\mathcal{A}_{3}$. Let $\mathcal{L}=\left\{x \in V^{\mathrm{ss}}(k) \mid k(x) \in \mathcal{A}_{n}^{\prime}\right\}$. The global zeta function for $\Phi=\prod_{v} \Phi_{v}$ in the Schwartz-Bruhat space $\mathscr{S}(V(\mathbb{A}))$ is defined by

$$
Z(\Phi, s)=\int_{G(\mathbb{A}) / G(k)}|\chi(g)|_{\mathbb{A}}^{2 s} \sum_{x \in \mathcal{L}} \Phi(g x) d g .
$$

It is proved by H. Saito [Sa03] that this integral converges absolutely for sufficiently large $\operatorname{Re}(s)$. For $L \in \mathcal{A}_{n}$, let $V_{k, L}=\left\{x \in V^{\mathrm{ss}}(k) \mid k(x)=L\right\}$. Then since $\mathcal{L}=\coprod_{L \in \mathcal{A}_{n}^{\prime}} V_{k, L}$ we have

$$
\begin{aligned}
Z(\Phi, s) & =\sum_{L \in \mathcal{A}_{n}^{\prime}} Z(\Phi, s, L), \\
Z(\Phi, s, L) & =\int_{G(\mathbb{A}) / G(k)}|\chi(g)|_{\mathbb{A}}^{2 s} \sum_{x \in V_{k}, L} \Phi(g x) d g .
\end{aligned}
$$

If $(G, V)$ is of the $D_{5}$ type, $V_{k, L}$ is a single $G(k)$-orbit. Also since the first Galois cohomology of $G_{x}^{\circ}$ vanishes for any local field of $k$, we have $G(\mathbb{A}) / G_{x}^{\circ}(\mathbb{A})=\left(G / G_{x}^{\circ}\right)(\mathbb{A})$. These combined with the usual unfolding method show that $Z(\Phi, s, L)$ has an Euler product and each Euler factor is what is called the local zeta function. H. Saito [Sa99] proved that even if $G(\mathbb{A}) / G_{x}^{\circ}(\mathbb{A}) \neq$ $\left(G / G_{x}^{\circ}\right)(\mathbb{A})$ for some $x \in V^{\mathrm{ss}}(k)$, the global zeta function $Z(\Phi, s)$ has a formula expressed via local zeta functions. Let $(G, V)$ be of the $E_{7}$ type. If $k$ has a real place, then $G(\mathbb{A}) / G_{x}^{\circ}(\mathbb{A}) \neq$ $\left(G / G_{x}^{\circ}\right)(\mathbb{A})$ for certain $x \in V^{\mathrm{ss}}(k)$. But the decomposition $\mathcal{L}=\coprod_{L \in \mathcal{A}_{n}^{\prime}} V_{k, L}$ is according to $\mathrm{H}^{1}\left(k, G_{x} / G_{x}^{\circ}\right)$ for an element $x \in V^{\mathrm{ss}}(k)$. Hence his result shows that $Z(\Phi, s, L)$ is a finite number of functions where each of them has an Euler product, and each Euler factor is a finite sum of local zeta functions. 


\section{Parameterizations of Reducible algebras for Cubic Cases}

In [WY92], Wright-Yukie showed that the semi-stable rational orbits $G(k) \backslash V^{\mathrm{ss}}(k)$ of the following three prehomogeneous vector spaces

$$
\begin{array}{lll}
\text { (1) } G=\mathrm{GL}_{1} \times \mathrm{GL}_{2}, & V=\mathrm{Sym}^{3} k^{2}, & \left(G_{2} \text { type }\right) \\
(2) \quad G=\mathrm{GL}_{3} \times \mathrm{GL}_{3} \times \mathrm{GL}_{2}, & V=k^{3} \otimes k^{3} \otimes k^{2}, & \left(E_{6} \text { type }\right) \\
\text { (3) } G=\mathrm{GL}_{6} \times \mathrm{GL}_{2}, & V=\wedge^{2} k^{6} \otimes k^{2}, & \left(E_{7} \text { type }\right)
\end{array}
$$

parameterize separable cubic algebras $\mathcal{A}_{3}$ of $k$. Let $\mathcal{A}_{3}=\mathcal{E}_{3} \amalg \mathcal{E}_{1,2} \amalg \mathcal{E}_{1,1,1}$, where

$$
\begin{aligned}
\mathcal{E}_{3} & =\{L \mid L / k \text { is a cubic field extension }\}, \\
\mathcal{E}_{1,2} & =\{L=k \times F \mid F / k \text { is a quadratic field extension }\}, \\
\mathcal{E}_{1,1,1} & =\{k \times k \times k\} .
\end{aligned}
$$

From number theoretical interests, we often would like to know about the subset $\mathcal{E}_{3}$ rather than the whole set $\mathcal{A}_{3}$ itself. Let $\mathrm{B}_{2} \subset \mathrm{GL}_{2}$ denote the set of lower triangular matrices. For the classical case (1), Shintani [Sh75] introduced the representation $\left(\mathrm{B}_{2}, \mathrm{Sym}^{2} k^{2}\right)$ to parameterize $\mathcal{E}_{1,2} \amalg \mathcal{E}_{1,1,1}$, and found a good information on $\mathcal{E}_{3}$. Shintani considered $\mathbb{Z}$-orbits and his method was recently extended to over a Dedekind domain by the author [T06]. In this section, we find analogous representation parameterizing $\mathcal{A}_{3} \backslash \mathcal{E}_{3}$ for (2), (3) and their $k$-forms over fields. The study of integral orbits will be important. Also similar problems should arise for the quartic case $\left(\mathrm{GL}_{3} \times \mathrm{GL}_{2}, \mathrm{Sym}^{2} k^{3} \otimes k^{2}\right)$ and quintic case $\left(\mathrm{GL}_{5} \times \mathrm{GL}_{4}, \wedge^{2} k^{5} \otimes k^{4}\right)$. These would be answered in the future.

For a quaternion algebra $\mathcal{B}$ of $k$ we keep the notation in Section 3 . Let $F$ be a quadratic field extension of $k$. We denote by $\sigma$ the generator of $\operatorname{Gal}(F / k)$. Let $\mathrm{N}_{F / k}$ be the norm map. Let $\mathrm{H}_{3}(F)=\left\{x \in \mathrm{M}_{3}(F) \mid{ }^{t} x=x^{\sigma}\right\}$. The group $\mathrm{GL}_{3}(F)$ acts on this space via $(g, x) \mapsto g x g^{\sigma}$, for $g \in \mathrm{GL}_{3}(F), x \in \mathrm{H}_{3}(F)$.

We define and discuss the three spaces in parallel; they will be distinguished as Cases (a), (b) and (c). Let

$$
\begin{array}{ll}
G_{1}=\mathrm{GL}_{3}(k) \times \mathrm{GL}_{3}(k), & Y=\mathrm{M}_{3}(k) \quad \text { in Case (a), } \\
G_{1}=\mathrm{GL}_{3}(F), & Y=\mathrm{H}_{3}(F) \quad \text { in Case (b), } \\
G_{1}=\mathrm{GL}_{3}(\mathcal{B}), & Y=\mathrm{H}_{3}(\mathcal{B}) \quad \text { in Case (c), }
\end{array}
$$

and put

$$
G=G_{1} \times \mathrm{GL}_{2}(k), \quad V=Y \oplus Y .
$$

(We already defined and considered Case (c) in Section 3. For later conveniences we do not admit $F$ to be $k \times k$ and treat Cases (a) and (b) separately.) We regard $G$ as an algebraic group over $k$ and $V$ as a variety over $k$. We write elements of $G$ as $g=\left(g_{11}, g_{12}, g_{2}\right) \in G$ in Case (a) and $g=\left(g_{1}, g_{2}\right)$ in Cases (b) and (c). We identify elements $x=\left(x_{1}, x_{2}\right) \in V$ with the 3 by 3 matrices $x(v)=x_{1} v_{1}+x_{2} v_{2}$ of linear forms in the variables $v_{1}$ and $v_{2}$, which we collect into the row vector $v=\left(v_{1}, v_{2}\right)$. With this identification, we define a rational action of $G$ on $V$ via

$$
(g \cdot x)(v)= \begin{cases}g_{11} x\left(v g_{2}\right)^{t} g_{12} & \text { in Case (a), } \\ g_{1} x\left(v g_{2}\right)^{t} g_{1}^{\sigma} & \text { in Case (b), } \\ g_{1} x\left(v g_{2}\right) g_{1}^{\iota} & \text { in Case (c), }\end{cases}
$$

Let $F_{x}(v)=\operatorname{det}(x(v))$ in Cases (a), (b) and $F_{x}(v)=\operatorname{Pfaff}_{3}(x(v))$ in Case (c). Let $P(x)$ be the discriminant of the binary cubic form $F_{x}(v)$. Then $P \in k[V]$ is a homogeneous polynomial of degree 12 and $P(g \cdot x)=\chi(g)^{2} P(x)$ for $g \in G, x \in V$ where

$$
\chi(g)= \begin{cases}\operatorname{det}\left(g_{1}\right)^{2} \operatorname{det}\left(g_{2}\right)^{2} \operatorname{det}\left(g_{3}\right)^{3} & \text { in Case (a), } \\ \mathrm{N}_{F / k}\left(\operatorname{det}\left(g_{1}\right)\right)^{2} \operatorname{det}\left(g_{2}\right)^{3} & \text { in Case (b), } \\ \mathcal{N}\left(g_{1}\right)^{2} \operatorname{det}\left(g_{2}\right)^{3} & \text { in Case (c). }\end{cases}
$$


Let $V^{\text {ss }}=\{x \in V \mid P(x) \neq 0\}$.

Definition 4.1. For $x \in V^{\mathrm{ss}}(k)$, we define

$$
Z_{x}=\operatorname{Proj} k\left[v_{1}, v_{2}\right] /\left(F_{x}(v)\right), \quad L_{x}=\Gamma\left(Z_{x}, \mathcal{O}_{Z_{x}}\right) .
$$

We define

$$
\begin{aligned}
& V^{(3)}=\left\{x \in V^{\mathrm{ss}}(k) \mid L_{x} \in \mathcal{E}_{3}\right\}, \\
& V^{(2)}=\left\{x \in V^{\mathrm{ss}}(k) \mid L_{x} \in \mathcal{E}_{1,2}\right\}, \\
& V^{(1)}=\left\{x \in V^{\mathrm{ss}}(k) \mid L_{x} \in \mathcal{E}_{1,1,1}\right\}
\end{aligned}
$$

The algebra $L_{x} \in \mathcal{A}_{3}$ depends only on $G(k)$-orbit of $x$. Hence each $V^{(i)}$ is a $G(k)$-invariant subset of $V^{\mathrm{ss}}(k)$.

For $A=k, F, \mathcal{B}$, we put

$$
\mathrm{P}_{1,2}(A)=\left\{\left(\begin{array}{ccc}
* & 0 & 0 \\
* & * & * \\
* & * & *
\end{array}\right) \in \mathrm{GL}_{3}(A)\right\} .
$$

Let $\mathrm{B}_{2} \subset \mathrm{GL}_{2}$ be the set of lower triangular matrices. We define

$$
P= \begin{cases}\mathrm{P}_{1,2}(k) \times \mathrm{P}_{1,2}(k) \times \mathrm{B}_{2}(k) & \text { in Case }(\mathrm{a}), \\ \mathrm{P}_{1,2}(F) \times \mathrm{B}_{2}(k) & \text { in Case }(\mathrm{b}), \\ \mathrm{P}_{1,2}(\mathcal{B}) \times \mathrm{B}_{2}(k) & \text { in Case }(\mathrm{c}) .\end{cases}
$$

We regard $P$ as an algebraic group over $k$, then is a parabolic subgroup of $G$. For $Y=\mathrm{M}_{3}(k)$, $\mathrm{H}_{3}(F)$ or $\mathrm{H}_{3}(\mathcal{B})$, we put

$$
Z=\left\{\left(\begin{array}{lll}
0 & 0 & 0 \\
0 & * & * \\
0 & * & *
\end{array}\right) \in Y\right\}
$$

and define $W=Z \oplus Y \subset V$. Then $W$ is a $P$-invariant subspace and $(P, W)$ is a prehomogeneous vector space with $W^{\text {ss }}=W \cap V^{\text {ss }}$ a single orbit over $\bar{k}$. We put $W^{(i)}=W^{\text {ss }}(k) \cap V^{(i)}$ for $i=1,2$. We note that for $w \in W^{\mathrm{ss}}, F_{w}(v)$ has a linear factor $v_{2}$ and hence $W^{\mathrm{ss}}(k) \cap V^{(3)}=\emptyset$.

We first consider the relation between $V^{(2)}$ and $W^{(2)}$.

Proposition 4.2. We have

$$
V^{(2)}=G(k) \times_{P(k)} W^{(2)} .
$$

Proof. In Case (c), we show in (2) of Theorem 3.17 that any orbit in $V^{(2)}$ has an element in $W^{\text {ss }}(k)$ and hence $V^{(2)}=G(k) \cdot W^{(2)}$. We did not consider Cases (a) and (b) in Section 3 but exactly the same arguments works and we can see any orbit in $V^{(2)}$ has an element in $W^{\mathrm{ss}}(k)$. Hence again we have $V^{(2)}=G(k) \cdot W^{(2)}$. The rest of arguments are also parallel for Cases (a), (b) and (c), and we write proofs for Case (c).

It is obvious that $P(k)$ fixes the set $W^{(2)}$. Let $w \in W^{(2)}, g=\left(g_{1}, g_{2}\right) \in G(k)$ and $x=g \cdot w \in$ $W^{(2)}$. We will show $g \in P(k)$. We first show $g_{2} \in \mathrm{B}_{2}(k)$. Since $x=g \cdot w, P_{x}(v)$ and $P_{w}\left(v g_{2}\right)$ coincide up to a constant multiple. On the other side since $w \in W^{(2)}, P_{w}(v)$ is a product of $v_{2}$ and an irreducible quadratic polynomial in $v$, and $P_{x}(v)$ is also. Hence $g_{2} \in \mathrm{GL}_{2}(k)$ must fix $(1: 0) \in \mathbb{P}^{1}(k)$ and consequently $g_{2} \in \mathrm{B}_{2}(k)$.

Let

$$
w=\left(\begin{array}{c|c}
0 & 0 \\
\hline 0 & w_{122}
\end{array}\right),\left(\begin{array}{c|c}
w_{211} & w_{212} \\
\hline w_{221} & w_{222}
\end{array}\right), \quad w_{122}, w_{222} \in \mathrm{H}_{2}(\mathcal{B}), w_{211} \in k,
$$

be the block representation of $w=\left(w_{1}, w_{2}\right)$. Then the coefficients of $v_{1}^{2} v_{2}$ of $F_{w}(v)$ is $w_{211} \mathrm{Pfaff}_{2}\left(w_{122}\right)$ and this does not vanish since $w \in W^{\mathrm{ss}}(k)$. Hence $w_{122}$ is invertible i.e., $w_{122} \in \mathrm{GL}_{2}(\mathcal{B})$. We consider the similar block representation for $x$, and we have $x_{122} \in$ 
$\mathrm{GL}_{2}(\mathcal{B})$. Let $g_{2}=\left(\begin{array}{ll}a & 0 \\ b & c\end{array}\right), p=a g_{1}, q=\left(g_{1}^{\iota}\right)^{-1}$. Then since $x_{1}=a g_{1} w_{1} g_{1}^{\iota}$ we have $x_{1} q=p w_{1}$. Write $p, q \in \mathrm{GL}_{3}(\mathcal{B})$ in the block form

$$
p=\left(\begin{array}{l|l}
p_{1} & p_{2} \\
\hline p_{3} & p_{4}
\end{array}\right), \quad q=\left(\begin{array}{l|l}
q_{1} & q_{2} \\
\hline q_{3} & q_{4}
\end{array}\right), \quad\left(p_{1}, q_{1} \in \mathcal{B}, p_{4}, q_{4} \in \mathrm{M}_{2}(\mathcal{B}) .\right)
$$

Then $p_{2} x_{122}=\left(\begin{array}{ll}0 & 0\end{array}\right)$ and $w_{122} q_{3}={ }^{t}\left(\begin{array}{ll}0 & 0\end{array}\right)$. Since $x_{122}, w_{122} \in \mathrm{GL}_{2}(\mathcal{B})$, we have $p_{2}=\left(\begin{array}{ll}0 & 0\end{array}\right)$, $q_{3}={ }^{t}\left(\begin{array}{ll}0 & 0)\end{array}\right.$. Thus we have $g=\left(g_{1}, g_{2}\right) \in P(k)$ and this finishes the proof.

We next consider the relation between $V^{(1)}$ and $W^{(1)}$. For $A=k, F, \mathcal{B}$, we put

$$
\mathrm{T}_{3}(A)=\left\{\left(\begin{array}{ccc}
* & 0 & 0 \\
0 & * & 0 \\
0 & 0 & *
\end{array}\right) \in \mathrm{GL}_{3}(A)\right\}
$$

Let $\mathrm{Z}_{2} \subset \mathrm{GL}_{2}$ be the center of $\mathrm{GL}_{2}$. We define

$$
H^{\circ}= \begin{cases}\mathrm{T}_{3}(k) \times \mathrm{T}_{3}(k) \times \mathrm{Z}_{2}(k) & \text { in Case (a), } \\ \mathrm{T}_{3}(F) \times \mathrm{Z}_{2}(k) & \text { in Case (b), } \\ \mathrm{T}_{3}(\mathcal{B}) \times \mathrm{Z}_{2}(k) & \text { in Case }(\mathrm{c}) .\end{cases}
$$

We regard $H^{\circ}$ as an algebraic group over $k$. We put

$$
\theta_{1}=\left(\begin{array}{lll}
0 & 0 & 1 \\
1 & 0 & 0 \\
0 & 1 & 0
\end{array}\right), \quad \theta_{2}=\left(\begin{array}{cc}
0 & 1 \\
-1 & -1
\end{array}\right), \quad \eta_{1}=\left(\begin{array}{lll}
1 & 0 & 0 \\
0 & 0 & 1 \\
0 & 1 & 0
\end{array}\right), \quad \eta_{2}=\left(\begin{array}{cc}
-1 & 0 \\
1 & 1
\end{array}\right)
$$

and define

$$
\theta=\left\{\begin{array}{ll}
\left(\theta_{1}, \theta_{1}, \theta_{2}\right) & \text { in Case (a), } \\
\left(\theta_{1}, \theta_{2}\right) & \text { in Case (b), } \\
\left(\theta_{1}, \theta_{2}\right) & \text { in Case (c), }
\end{array} \quad \eta= \begin{cases}\left(\eta_{1}, \eta_{1}, \eta_{2}\right) & \text { in Case (a) } \\
\left(\eta_{1}, \eta_{2}\right) & \text { in Case (b) } \\
\left(\eta_{1}, \eta_{2}\right) & \text { in Case (c) }\end{cases}\right.
$$

Let $\mathfrak{A}_{3}, C_{2}$ be respectively the group generated by $\theta$ and $\eta$, and $\mathfrak{S}_{3}$ the group generated by $\mathfrak{A}_{3}$ and $C_{2}$. Then $\mathfrak{S}_{3}$ is isomorphic to the symmetric group of degree $3, \mathfrak{A}_{3}$ to the alternating subgroup, and $\mathfrak{S}_{3}=\mathfrak{A}_{3} \rtimes C_{2}$. It is easy to see that $\mathfrak{S}_{3}$ normalizes $H^{\circ}$. We put $H=H^{\circ} \rtimes \mathfrak{S}_{3}$. Then $H^{\circ}$ is the identity component of $H$. We put

$$
U=\left\{\left(\left(\begin{array}{ccc}
0 & 0 & 0 \\
0 & b & 0 \\
0 & 0 & -c
\end{array}\right),\left(\begin{array}{ccc}
a & 0 & 0 \\
0 & -b & 0 \\
0 & 0 & 0
\end{array}\right)\right) \in V \mid a, b, c \in k\right\} .
$$

Then $U$ is a $H$-invariant subspace. We put $U^{\text {ss }}=U \cap V^{\text {ss }}$. For $u \in U, F_{u}$ coincides with $v_{1} v_{2}\left(v_{1}-v_{2}\right)$ up to constant multiple. Hence $U^{\mathrm{ss}}(k) \subset W^{(1)}$.

Proposition 4.3. We have

$$
\begin{aligned}
V^{(1)} & =G(k) \times_{H(k)} U^{\mathrm{ss}}(k), \\
W^{(1)} & =P(k) \times_{H(k) \cap P(k)} U^{\mathrm{ss}}(k) .
\end{aligned}
$$

Proof. Since the proofs are similar, we here write a proof for Case (c). Let us consider the first formula. We show in (1) of Theorem 3.17 that any orbit in $V^{(1)}$ has an element in $U^{\mathrm{ss}}(k)$ and hence $V^{(1)}=G(k) \cdot U^{\mathrm{ss}}(k)$. Let $x \in U^{\mathrm{ss}}(k), g=\left(g_{1}, g_{2}\right) \in G(k)$ and assume $y=g x \in U^{\mathrm{ss}}(k)$. Since the set of roots of $F_{x}(v)$ and $F_{y}(v)$ in $\mathbb{P}^{1}$ are both $\{(0: 1),(1: 0),(1: 1)\}, g_{2} \in \mathrm{GL}_{2}(k)$ fixes this set. Since the $\mathrm{GL}_{2}$-part of $\mathfrak{S}_{3}$ coincides with the permutation group of this set, there exists $\sigma=\left(\sigma_{1}, \sigma_{2}\right) \in \mathfrak{S}_{3}$ such that $\sigma_{2} g_{2} \in \mathrm{GL}_{2}(k)$ is a scalar matrix. Hence $\sigma g x \in U^{\mathrm{ss}}(k)$ and the $\mathrm{GL}_{2}$-component of $\sigma g$ is in $\mathrm{Z}_{2}(k)$. Now it is easy to see that $G_{1}$-component of $\sigma g$ is in $T_{3}(\mathcal{B})$. Hence $\sigma g \in H^{\circ}(k)$ and we have $g \in H(k)$. This proves the first formula. 
For the second formula, it is enough to show $W^{(1)}=P(k) \cdot U^{\mathrm{ss}}(k)$. Let $w \in W^{(1)}$ and

$$
w=\left(\left(\begin{array}{c|c}
0 & 0 \\
\hline 0 & w_{122}
\end{array}\right),\left(\begin{array}{l|l}
w_{211} & w_{212} \\
\hline w_{221} & w_{222}
\end{array}\right), \quad w_{122}, w_{222} \in \mathrm{H}_{2}(\mathcal{B}), w_{211} \in k,\right.
$$

be the block representation of $w=\left(w_{1}, w_{2}\right)$. We can prove $w \in P(k) \cdot U^{\mathrm{ss}}(k)$ by the following steps. Let $P_{1,2}(\mathcal{B})=L_{1,2}(\mathcal{B}) \ltimes U_{1,2}(\mathcal{B})$ be the Levi decomposition.

(1) Since $w_{211} \in k^{\times}$, by multiplying an element of $U_{1,2}(\mathcal{B})$ if necessary, we may assume $w_{212}=w_{221}=0$.

(2) Because of the result of $D_{5}$-type, by multiplying an element of $L_{1,2}(\mathcal{B})$ if necessary, we may further assume $w_{122}$ and $w_{222}$ are both diagonal.

(3) Moreover, by multiplying an element of $B_{2}(k)$, we may assume the set of roots of $F_{w}(v)$ is $\{(0: 1),(1: 0),(1: 1)\}$.

Under these conditions, either $w$ or $\eta w$ must be an element of $U^{\mathrm{ss}}(k)$. This finishes the proof.

\section{REFERENCES}

[KY97] A.C. Kable and A. Yukie. Prehomogeneous vector spaces and field extensions II. Invent. Math., 130:315344, 1997.

[Sa97] H. Saito. On a classification of prehomogeneous vector spaces over local and global fields. Journal of Algebra, 187:510-536, 1997.

[Sa99] H. Saito. Explicit form of the zeta functions of prehomogeneous vecotr spaces. Math. Ann., 315:587-615, 1999.

[Sa03] H. Saito. Convergence of the zeta functions of prehomogeneous vector spaces. Nagoya. Math. J., 170:131, 2003.

[Se65] J.P. Serre. Cohomologie galoisienne, volume 5 of Lecture Notes in Mathematics. Springer-Verlag, Berlin, Heidelberg, New York, 1965.

[Sh75] T. Shintani. On zeta-functions associated with vector spaces of quadratic forms. J. Fac. Sci. Univ. Tokyo, Sect IA, 22:25-66, 1975.

[T06] T. Taniguchi. Distributions of discriminants of cubic algebras. Preprint 2006, math.NT/0606109.

[T04] T. Taniguchi. On the zeta functions of prehomogeneous vector spaces for a pair of simple algebras. math.NT/0403253, to appear in Ann. Inst. Fourier.

[WY92] D.J. Wright and A. Yukie. Prehomogeneous vector spaces and field extensions. Invent. Math., 110:283314, 1992.

[Y97a] A. Yukie. A remark on the regularity of prehomogeneous vector spaces. Preprint, math.RT/9708209.

[Y97b] A. Yukie. Prehomogeneous vector spaces and field extensions III. J. Number Theory, 67:115-137, 1997.

Department of Mathematical Sciences, University of Tokyo

E-mail address: tani@ms.u-tokyo.ac.jp 